\title{
Gigabits XF Type Correlator
}

\author{
T. Hashimoto, K. Ozeki, \& K. Natori \\ Oki Electric Industry Co., Ltd., Tokyo, Japan
}

\begin{abstract}
The National Astronomical Observatory of Japan (NAO), has an Optical Linked VLBI Experiment Project named OLIVE that directly connects a correlation site wit h some other VLBI observation stations in network by optical fiber,and correlates in real time. 2 Gbps (1 Gsps 2-bit or 2 Gsps 1-bit) or 8 Gbps (4 Gsps 2-bit) VLBI correlator is necessary in OLIVE. We are developing Gigabits correlators (XF type) using special-purpose correlation chips developed by NAO. We have already completed the Ultra Wideband Correlator named UWBC (2 Gsps 2-bit 256 lags) of 15 baselines for the Nobeyama Millimater Array (NMA). The Nobeyama Radio Observatory (NRO) offered the common use of a new wide-band observation system to astronomers since March 1997 . We consider that the 2-Gbps VLBI correlator, which is needed in the OLIVE project, is possible to manufacture. It is also possible to manufacture a 8 Gbps-VLBI correlator using the UWBC chip.
\end{abstract}

\section{Introduction}

NAO has a new VLBI observation project (OLIVE) in collaborate with a common carrier. A correlation site and some observation stations are connected with ATM (Asynchronous transfer mode) networks $(2.4 \mathrm{Gbps})$ in real time. The OLIVE project advanced in 3 stages (VSOP, LEAF: Low temperature source AFirmative detection, and DOVE: Dark and compact Object Vlbi survEy) as follows. The equipment of the first stage has already been tested.

$\begin{array}{lcccc}\text { stage } & \text { year } & \text { network speed } & \text { throughput } & \text { stations } \\ \text { VSOP } & 1997- & 2.4 \mathrm{Gbps} & 256 \mathrm{Mbps} & 3 \\ \text { LEAF } & 1998- & 2.4 \mathrm{Gbps} & 2 \mathrm{Gbps} & 3 \\ \text { DOVE } & 2000- & 2.4 \mathrm{Gbps} \times \mathrm{4} \text { (TBD) } & 8 \mathrm{Gbps} & 5\end{array}$

We are developing Gigabits correlators (XF type) by the following steps using special-purpose correlation chips developed by NAO.

(1) We developed the prototype UWBC that has performance of 1 Gsps 1-bit $256 \mathrm{l}$ ags in 1994. The Near Real-time Fringe Detector (NRFD) which has performance of $256 \mathrm{Msps} 2$-bit 1024 lag was developed in VSOP project in 1995. (2) We developed the UWBC (15 baselines) which has performance of 2 Gsps 2-bit 256 lags for NMA from 1995 to 1997.

(3) We converted the prototype UWBC to 2 Gbps-VLBI correlator in 1996. Now, we are estimating converted correlator.

(4) We will propose the development of VLBI correlator that has performance of 1 Gsps 2-bit 256 lags using the UWBC chip. (1997:TBD)

(5) We will examine the expansion of the correlator that has a performance of 4 Gsps 2-bit 256 lags. (2000:TBD)

\section{Developed the NRFD and the UWBC}

A priori is calculated from the orbit of the HALCA by VSOP. The NRFD calculate an orbit of HALCA accurately always in nearly real time. It shall be correlated exactly by VSOP-FX correlator. The chip has not only a correlative function but also other functions (for example, delay tracking, fringe rotation). 
The NRFD detected signal from HALCA in real time for the first time on the 12th March 1997. It was concluded that the HALCA is active from this result. Characteristics of NRFD are,

(1) Various input data format can be processed

(2) Wide fringe frequency window; $80 \mathrm{~Hz}$ (maximum)

(3) Throughput: $256 \mathrm{Msps}$ 1-bit 1024 lags, $128 \mathrm{Msps} 2$-bit 2048 lags (maximum)

(4) Multi-baselines with only a box. (Without changing wiring, combination of baselines can be changed. 4 stations, 6 baseline and 2 auto correlation)

(5) It can be utilized on a spectrometer in an auto correlation mode.

We have constructed a wide-band correlator named UWBC (15 baselines) using two kinds of special-purpose LSI chips developed by NRO. The maximum signal processing speed is 2 -bit $\times 2$ Gsps, resulting in the frequency bandwidth of $2 \mathrm{GHz}$ in DSB mode. This is about 6 times wider than that obtained with previous $320 \mathrm{MHz}$ bandwidth system. Major specifications of UWBC are,

(1) Three observational modes are available (see below) and can be selected via commands from the host computer.

$\begin{array}{lccc}\text { sampling } & \text { clock frequency } & \text { number of complex lags } & \text { bandwidth } \\ 2 \text { Gsps-2-bit } & 32 \mathrm{MHz} & 256 \text { complex lags } & 1024 \mathrm{MHz} \\ 1 \text { Gsps-2-bit } & 32 \mathrm{MHz} & 512 \text { complex lags } & 512 \mathrm{MHz} \\ 512 \mathrm{Msps}-2 \text {-bit } & 16 \mathrm{MHz} & 512 \text { complex lags } & 256 \mathrm{MHz}\end{array}$

(2) Delay tracking function

(3) Phase rotation function: 0/90/180/270 degree switching

(4) Integration period (IP): $0.1,0.2,--, 0.9,1.0$ second

(5) Digital Signal Processors are mounted for the primary signal processing

\section{Converting the Prototype UWBC and Develop 8 Gbps-VLBI Cor- relator}

Gigabit VLBI recorder is now being developed by Communications Research Laboratory, Japan (CRL). To test GBR, a temporary correlator was prepared. UWBC chip has the functions which are necessary in the VLBI system. Before the UWBC was developed, we manufactured the prototype UWBC. The technical problems are (1) performance and functions of UWBC chip, (2) mount some chips that have many pins on board and (3) multi-layered board is adopted. The prototype UWBC was converted on VLBI correlator for GBR estimating. Now bit tracking and fringe rotation function, which are required in VLBI, are being tested.

We consider that there are no obstacles in the development of 8 Gbps correlator using the UWBC chip. (Ability: 32 lag per 1 chip) By connecting UWBC1 chip cascade, the number of lags which can be managed is enlarged. (32 lag/chip $\mathrm{X} 8 \mathrm{ch}$ ips $=256 \mathrm{lag}$ ) $\mathrm{XF}$ type correlator should improve throughput by increasing parallel stage from 2 stages(in UWBC) to 4 stages. ( 2 Gsps $\mathrm{X} 4$ parallel $=8$ Gsps) However, there are some technical problems in developing a 8 Gbps-VLBI correlator. In order to overcome problems, another technique will be investigated. 